\title{
Benchmarking of Power Quality Performance in Transmission Systems - CIGRE WG C4.27 Perspective
}

\author{
Jako Kilter \\ Department of Electrical Power Engineering \\ Tallinn University of Technology \\ Tallinn, Estonia \\ jako.kilter@ttu.ee \\ Sean Elphick \\ Australian Power Quality \& Reliability Centre \\ University of Wollongong \\ Wollongong, Australia \\ sean_elphick@uow.edu.au \\ John van Coller \\ School of Electrical and Information Engineering \\ University of the Witwatersrand \\ Johannesburg, South Africa \\ john.vancoller@wits.ac.za
}

\author{
Davor Vujatovic \\ VandA Engineering Ltd \\ London, UK \\ davor.vujatovic@vanda.uk.com
}

\author{
Herwig Renner \\ Institut für Elektriche Anlagen \\ Technische Universität Graz \\ Graz, Austria \\ herwig.renner@tugraz.at
}

Frans van Erp

Department of Asset Management

TenneT TSO BV

Arnhem, The Netherlands

frans.van.erp@tennet.eu

\begin{abstract}
This paper describes the activities of CIGRE WG C4.27 "Benchmarking of Power Quality Performance in Transmission Systems". The WG was established in December 2012 in recognition of demand for coherent set of guidelines for benchmarking power quality performance in existing and future transmission networks. During the work the members of the WG have compiled extensive material related to the state of the art of benchmarking power quality performance in transmission systems. This paper summarizes current understanding of benchmarking power quality performance issues and identifies directions in which the WG will continue to work in order to provide resolutions to outstanding questions.
\end{abstract}

Index Terms-Power quality, power quality measurements, power quality benchmarking, power quality parameters.

\section{INTRODUCTION}

Power quality benchmarking is receiving growing interest from both transmission companies and electricity regulators. The work described in this paper is aimed at developing guidelines for technical performance benchmarking by combining the input from specialists in CIGRE C4 (System Technical Performance) and C5 (Electricity Markets and Regulation). Technical performance here refers to interruption performance (reliability), availability, voltage dips, and voltage waveform quality (harmonics, unbalance, magnitude, and flicker). The work undertaken by CIGRE C4 [1] identified that various transmission companies are using widely different indices for technical performance reporting, especially in the case of voltage dips, system reliability, and availability reporting. Various commercial benchmarking companies also use very different indices, and in some cases the manner in which common indices are defined and applied can vary greatly - making compari- sons between performance reported by these different companies difficult. The peer groups for which such benchmarks are determined are also often not well selected, and characteristics of the systems are often not included with the benchmarking information. Furthermore, the identification of data that should be excluded from analysis is not consistent among transmission companies, again making comparisons difficult (e.g. third party caused incidents).

The scope of the WG is to develop a set of guidelines for transmission technical performance benchmarking based on:

- A review of the practical application and definitions of the indices proposed by the CIGRE WG C4.07 [1] for Transmission and Sub-Transmission systems (this report on Power Quality Indices and Objectives has provided an in-depth analysis of possible indices, and provides proposals on indices and the manner in which these indices are calculated). These proposed indices may be used as a starting point, but may well need to be adapted or further defined for application to benchmarking.

- The definition of important "internal" indices such as circuit availability and line fault rates.

- Consideration of the implications of recommendations on monitoring and reporting systems [2].

- Recommendations on how systems can be described (important characteristics to be identified as part of the benchmarking information) to assist transmission operators, regulators, and benchmarking companies in the identification of a suitable peer group.

- Highlight what historical data, system descriptions, and calculation methodologies need to be in place for effective benchmarking to be undertaken (or alterna- 
tively highlight cases where benchmarking is not an appropriate tool for decision making).

For clarity, the aim of this working group is not to actually undertake benchmarking; rather, to provide a practical framework for benchmarking studies.

Although the focus of the proposed guideline is on assessing the relative technical performance of two comparable systems, economic issues are also taken into consideration as the technical link between economic and technical benchmarks is important to regulators and the companies they regulate.

The paper is divided into six sections as follows: In section two PQ performance objectives are covered. Section three provides a brief summary of the existing indices typically used in system power quality benchmarking. A summary of recommended methods and indices for system benchmarking is given in section four. Section five gives an overview of system characteristics influencing PQ performance benchmarking. The paper concludes with conclusions and discussion.

\section{OBJECTIVES OF BENCHMARKING PQ PERFORMANCE}

Benchmarking is above all the comparison between one set of (PQ) performance measures against another. A comparison (benchmarking) between the performance of different systems does not imply any value judgement. For any index of the PQ performance, one system will give a different result from another for many reasons including:

- characteristics of the system;

- design of the network;

- climate conditions;

- characteristics of the connected customers;

- applicable standards;

- $\quad$ funding available to the utility;

The typical steps in the power quality benchmarking process are:

1. Select benchmarking indices;

2. Collect power quality data;

3. Select the benchmark;

4. Determine target performance levels.

The benchmarking process therefore starts with selection of indices suitable for the purpose of describing the system performance in terms of power quality over a given period of time.

\section{EXISTING INDICES}

\section{A. Harmonics}

For harmonics assessment, statistical evaluation is used to produce a number of indices based on measured data. The majority of standards (including IEEE) that specify statistical evaluation methods and limits for harmonics now employ the measurement methods described in IEC 61000-4-7 [3] and IEC 61000-4-30 [4]. Common statistical indicators used include $99^{\text {th }}$ percentile and $95^{\text {th }}$ percentile values. Common assessment intervals include 3 second and 10 minute values which are evaluated over daily and weekly evaluation periods. In all cases, statistical evaluation is used to determine an index for each harmonic order (plus THD) which is then compared to a limit value.

For transmission systems, the two most commonly applied or referenced standards which specify limits (or planning levels) for harmonics are IEEE 519 [5] and IEC 61000-3-6 [6] (there are national standards, such as G5/4-1 in the UK [7] or jurisdictional clones such as the Australian equivalent TR IEC 61000.3.6:2012 [8]). These standards specify limits or planning levels for harmonic up to the $50^{\text {th }}$ order. As such, the existing indices for harmonics are generally one or more statistical measures (e.g. $99^{\text {th }}$ percentile, $95^{\text {th }}$ percentile) of harmonic data measured over one or more time intervals (e.g. 3 second, 10 minute) for 50 orders plus THD evaluated over one or more evaluation intervals (e.g. one day, one week) for each phase. It should be noted that there are other standards available and in use, but none apply directly to the transmission systems. These standards are applied to low voltage systems or medium and high voltage distribution systems.

Other than a requirement for consistent evaluation methodologies, the current widely used standards for either harmonic measurement methodology or application of limits and planning levels or are effectively silent with regard to guidelines for benchmarking. One resources that does provide guidance for benchmarking of harmonics is the CEER Guidelines of Good Practice on the Implementation and Use of Voltage Quality Monitoring Systems for Regulatory Purposes [9]. The proposed benchmarking methodology for voltage harmonics as described in the document is reproduced below:

- The following harmonics orders are considered: -Harmonic subgroup 3 (in LV networks only); -Harmonic subgroup 5;

-Harmonic subgroup 7;

-The total harmonic distortion over all odd harmonic subgroups of order 9 through 39 ;

-The total harmonic distortion over all even harmonic subgroups of order 2 through 40.

- The following indices are calculated for each site:

-The $99 \%$ value of the harmonic characteristic over one year;

-For harmonic subgroups 3, 5 and 7: The number of values that exceed the EN50160 values during the year;

-For harmonic subgroups 3,5 and 7: The number of values that exceed $75 \%$ of the EN 50160 values during the year;

-The number of 10-minute intervals during which at least one of the EN 50160 limits is exceeded.

- The following methods are used to calculate system indices (i.e. a single number for a large number of sites or the entire network):

-The $95 \%$ value of the $99 \%$ values for each site;

-For harmonic subgroups 3, 5 and 7: The 95\% value of the number of values that exceed the EN 50160 limits; -For harmonic subgroups 3, 5 and 7: The $95 \%$ value of the number of values that exceed $75 \%$ of the EN 50160 limits;

-For harmonic subgroups 3, 5 and 7: The percentage of sites for which at least one of the values exceeds the EN 50160 limits;

-For harmonic subgroups 3, 5 and 7: The percentage of sites for which at least one of the values exceeds $75 \%$ of the EN 50160 limits;

-The $95 \%$ value of the number of 10 -minute intervals during which at least one of the EN 50160 limits is exceeded.

Even if the most common evaluation methods for measurement and evaluation of harmonic performance are used, there will be a very large number of individual harmonic indices produced by any survey. The following is a common set of site indices:

- 50 harmonic orders plus THD; 
- 3 phases;

- $\quad 99^{\text {th }}$ percentile daily 3 second values;

- $\quad 95^{\text {th }}$ percentile weekly 10 minute values.

Calculation of the above will result in over 300 indices for each site; far more than are required for benchmarking purposes. As such, there is a requirement to select a subset of harmonic indices for the purposes of benchmarking against peers.

The first step in the benchmarking process is to calculate indices for each site. These site indices can then be used to calculate indices for multiple sites or for the entire network. Calculation of site indices is achieved by applying a statistical measure to each harmonic order included in the data subset over the evaluation period. Before calculation of site indices, it is recommended that flagged data (as defined in IEC 61000-430) be removed.

For the purposes of benchmarking it is recommended that the $95^{\text {th }}$ percentile value for each harmonic order in the data subset be used as the site index. Specifically, it is proposed that the following should be calculated at each site and used for benchmarking:

- The $95^{\text {th }}$ percentile value of the THD of all harmonic orders over the evaluation period;

- The $95^{\text {th }}$ percentile value of the THD of all orders (odd and even) $2^{\text {nd }}$ to $15^{\text {th }}$;

- $\quad$ The $95^{\text {th }}$ percentile value of the THD of all orders (odd and even) $15^{\text {th }}$ to $50^{\text {th }}$.

In addition to the above the transmission network operator may also choose to calculate the following:

- The $95^{\text {th }}$ percentile value of the THD of all orders (odd and even) $50^{\text {th }}$ to $100^{\text {th }}$.

For sites with multiple phases, the largest index across all three phases should be retained as the benchmarking index.

\section{B. Flicker}

Voltage fluctuations and flicker have been assessed for many years using the internationally-recognized concept of a 10-min short-term flicker severity $\left(\mathrm{P}_{\mathrm{st}}\right)$ and a 120-min longterm flicker severity $\left(\mathrm{P}_{\mathrm{lt}}\right)$ as defined in IEC 61000-4-15 [10]. The American standard IEEE P1453 [11] refers to the same method.

In the Far East (Japan, Korea, Taiwan) the Japanese $\Delta$ V10 -method [12] is widely used. In this case values are determined on a minute-by-minute basis. The relevant value is the $4^{\text {th }}$ highest after one hour.

Due to flicker transfer characteristics (decrease of flicker with increasing short circuit capacity) and the cubic summation law, flicker in transmission systems is usually dominated by single large installations like electric arc furnaces. Comparative measurements between $\mathrm{P}_{\text {st } \max }$ values and $\Delta \mathrm{V} 10\left(4^{\text {th }}\right)$ give a ratio of 3 for those applications [15].

For MV and HV IEC does not provide compatibility levels, only planning levels. Indicative values are provided by IEC 61000-3-7 [16], IEEE 1453 refers to the same standard.

The $95 \%$ and $99 \%$ probability weekly value of $\mathrm{P}_{\text {st }}$ and the $95 \%$ probability weekly value of $\mathrm{P}_{1 \mathrm{t}}$ should be provided by measurement. The weekly $95 \%$ probability value of $\mathrm{P}_{\mathrm{st}}$ and $\mathrm{P}_{\mathrm{lt}}$ should not exceed the chosen planning levels. The $99 \%$ probability value of $\mathrm{P}_{\text {st }}$ may exceed the planning level by a factor (for example: 1 to 1,5 ) to be specified by the system operator or owner.

In [1] it is recommends to use the weekly $95 \%$ probability value of $\mathrm{P}_{\mathrm{lt}}$ as site index, but does not consider a system index as essential.

\section{Unbalance}

Voltage unbalance is commonly assessed based on the ratio of negative phase sequence component of the supply voltage to positive phase sequence component. In some cases the ratio of zero-sequence to positive sequence is also used. Voltage unbalance measurement and evaluation procedure is defined in detail in IEC 61000-4-30 [4], whereas IEC 61000-3-13 [17] provides planning levels. According to [1] only fundamental components shall be used, i.e. all harmonic components should be eliminated. For assessment different values can be used, for example, 10-cycle $(50 \mathrm{~Hz})$ and 12-cycle $(60 \mathrm{~Hz}), 3$-second intervals and 10-minute intervals. For unbalance 2-hour values (obtained by combining 10-minute values) can also be used.

According to the CEER Guidelines [9], 10-minute ratio of negative and positive voltage should be calculated and flagged values due to interruptions, voltage dips, voltage swells, rapid voltage changes, voltage transients and transient overvoltages should be removed from the statistics. For every monitor location, the following site indices should be calculated over each calendar year:

- The $99 \%$ value of the unbalance over one year;

- The number of unbalance values that exceed $2 \%$ during the year;

- The number of unbalance values that exceed $1.5 \%$ during the year.

For a system with at least 20 monitor locations, the following system indices should be calculated for every calendar year:

- $\quad$ The $95 \%$ value of the $95 \%$ values for each site;

- The $95 \%$ value of the number of unbalance values that exceed $2 \%$;

- $\quad$ The $95 \%$ value of the number of unbalance values that exceed $1.5 \%$;

- The percentage of sites for which at least one of the unbalance values exceeds $2 \%$;

- The percentage of sites for which at least one of the unbalance values exceeds $1.5 \%$.

In [1], it is recommended to use consistent index for characterizing voltage unbalance, e.g., the $95 \%$ probability weekly value of Unegsh. (negative sequence voltage unbalance factor). The recommended system index is the percentage of site indices that exceeds the voltage characteristics in a given reporting period. The choice of a specific objective to be met is a matter of agreement between the system operator and the regulator. With regard to planning level it is recommended in [1] to compare the actual level of voltage unbalance with the planning level by using one or both of the following:

- The $95 \%$ probability daily value of Uneg,vs (r.m.s. value of voltage unbalance factor over 'very short' 3-s periods);

- The $99 \%$ probability weekly value of Uneg,sh (rms value of voltage unbalance factor over 'short' $10 \mathrm{~min}$. periods).

The $95 \%$ probability value should not exceed the planning level. For the $99 \%$ probability indices, planning levels may be exceeded by a factor, e.g. 1.25-2 times, to be specified by the system operator, depending on the system and load characteristics.

\section{Voltage Dips and Swells}

Various methods of indices to characterise voltage dips and various methods of reporting dips are proposed by different 
organisations. No single method has been accepted among organisations, though various methods show similarities.

Indices for individual voltage dips include:

- Voltage dips according to IEC 61000-4-30: -characterizes single-event voltage dips in terms of magnitude and duration;

- Voltage sag indices according to IEEE P1564: -characterizes single-event voltage dips in terms of magnitude and duration;

-voltage-dip energy;

-voltage-sag severity;

- Voltage swells are recorded in a similar way to the recording of voltage dips.

Methods of reporting dip results for a site or for a network (system) are as follows:

- Magnitude-duration table in form of the so-called density table is in common use where the columns of the table represent ranges of voltage-dip duration and the rows represent ranges of retained voltage. Various table formats are given in IEC TR 61000-2-8, IEEE P1564, EN50160, NRS-048-2, Unipede DISDIP.

- $\quad$ SARFI indices according to IEEE P1564:

-System Average RMS variation Frequency Index $\left(\mathrm{SARFI}_{\mathrm{x}}\right)$ gives the number of events per year with a duration between 0.5 cycle and 1 minute and a retained voltage less than $\mathrm{X} \%$ and is obtained as a weighted average over all monitor locations within a supply network or within part of the supply network, however the term is also used to refer to the event frequency at one location;

-System Instantaneous Average RMS (Variation) Frequency Index voltage (SIARFIx), System Momentary Average RMS (Variation) Frequency Index voltage (SMARFIx), System Temporary Average RMS (Variation) Frequency Index voltage (STARFIx), represents the average number of specified instantaneous rms variation measurement events that occurred over the assessment period per customer served. The specified disturbances are those with a magnitude less than $\mathrm{x}$ for sags or a magnitude greater than $\mathrm{x}$ for swells and duration in the range of respectively: 0.5 - 30 cycles, 30 cycles to 3 seconds, and 3 - 60 seconds for sags and swells.

- Voltage-sag co-ordination chart gives the number of events per year, sags and interruptions, as a function of the severity of the event, and these charts are described in IEEE Std.493 and IEEE Std.1346;

- Dip Performance/Sensitivity Area Plots describes specific areas on a magnitude/duration plane that attempt to provide generalized guidelines on areas where dips are likely to occur, and areas that customers are likely to be affected by, in order to reduce the number of indices that need to be reported and managed, based on the most "appropriate" grouping of dip events; plots are given in:

-IEEE P1564 SARFI-Curve gives the number of events per year with a duration between 0,5 cycle and 1 minute below the predefined curve;

-NRS-048-2 dip reporting method;

-EDF dip reporting method used in contracts.

All dip indices make use of the individual dip measurement methods described in IEC 61000-4-30. The dip is characterised by the residual voltage and its duration. The dip threshold and the hysteresis voltage are both set by the user. Regarding the dip evaluation, polyphase aggregation and time aggregation is applied. Voltage dips classification is performed with the aid of magnitude-duration tables for a specified period and is used in all standards on the networks of electricity systems. The cell format of the magnitude-duration table is customised to the needs of the users of the standards.

\section{E. Interruptions}

The most common indices used for benchmarking are the System Minute (SM) and the System Energy Not Served (ENS) Factor:

The System Minute is calculated from the quotient Estimated energy not supplied (MWh) $\times 60$ System annual maximum demand (MW)

The System Energy Not Served (ENS) Factor is calculated from the quotient

\section{Estimated energy not supplied (MWh)}

$\overline{\text { System total annual energy supplied (MWh) }}$

For generators, the corresponding indices are System Minutes (SM) (imported)

$$
\frac{\text { Estimated energy not imported (MWh) } \times 60}{\text { System annual maximum demand (MW) }}
$$

and Estimated Energy Not Imported (ENI)

$$
\frac{\text { Estimated energy not imported(MWh) }}{\text { System total annual energy supplied (MWh) }}
$$

Other indices that do not take the effect on the customer into account are more concerned about loss of service between the transmission company and the distribution company (this interface is defined as the Connection Point).

A transmission system index that quantifies the number of connection point interruptions over the reporting period is SAIFI (System Average Interruption Frequency Index), which is measured in interruptions per connection point during the reporting period. Another transmission system index that quantifies the duration of the Connection Point interruptions is SAIDI (System Average Interruption Duration Index), which is measured in hours of interruptions per reporting period. If no customers experience an interruption due to a particular Connection Point interruption then this interruption may be excluded from the calculation of the above indices.

\section{RECOMMENDED METHODS AND INDICES FOR SYSTEM BENCHMARKING}

If a single value is required for a large number of sites or even an entire network, it is recommended that the $95^{\text {th }}$ percentile value of the site indices defined above be used as the index to be benchmarked. This $95^{\text {th }}$ percentile value is then termed the system index. The $95^{\text {th }}$ percentile value is selected as it is specified in the CEER Guidelines of Good Practice on the Implementation and Use of Voltage Quality Monitoring Systems for Regulatory Purposes and also in IEC/TR 61000-3-6, -7 and -13 as follows:

"Compatibility levels are generally based on the $95 \%$ probability levels of entire systems, using statistical distributions which represent both time and space variations of disturbances. There is allowance for the fact that the system operator or owner cannot control all points of a system at all times. 
Therefore, evaluation with respect to compatibility levels should be made on a system-wide basis and no assessment method is provided for evaluation at a specific location."

However, careful consideration of the number and location of monitored sites is necessary. The CEER guideline recommends performing monitoring at all EHV/HV, EHV/MV and HV/MV substations.

The benchmarking evaluation period is the time interval over which index calculation will be performed. Many standards define a one week evaluation interval as being appropriate to determine the characteristics harmonic performance at a site. However, recent work performed within the WG suggests that a one week interval is insufficient to fully characterise the performance of a site. Determination of an appropriate evaluation interval for calculation benchmarking indices is an area of ongoing work for the WG.

Based on the above, specifically, the following should be calculated:

\section{A. Harmonics}

- The $95^{\text {th }}$ percentile value of all site indices for the THD of all harmonic orders;

- The $95^{\text {th }}$ percentile value of all sites indices for the THD of all orders (odd and even) $2^{\text {nd }}$ to $15^{\text {th }}$;

- The $95^{\text {th }}$ percentile value of all site indices for the THD of all orders (odd and even) $15^{\text {th }}$ to $50^{\text {th }}$.

In addition to the above the transmission network operator may also choose to calculate the following:

- The $95^{\text {th }}$ percentile value of all site indices for the THD of all orders (odd and even) $50^{\text {th }}$ to $100^{\text {th }}$.

\section{B. Flicker}

- The $95^{\text {th }}$ percentile value of all site indices for the $\mathrm{P}_{\mathrm{st}}$ and $\mathrm{P}_{\mathrm{lt}}$

\section{Unbalance}

- The $95^{\text {th }}$ percentile value of all site indices for the Uneg,sh

\section{Voltage Dips and Swells}

Various methods of indices to characterise voltage dips and various methods of reporting dips are proposed by different organisations. No single method has been accepted among organisations, though various indices and its used methods to construct the indices show similarities. Ongoing work in the WG will concentrate on proposing relevant system index(es) for benchmarking.

\section{E. Interruptions}

Because of the difficultly of applying the Connection Point Indices (with potential back-feeding, loss of a connection point does not necessarily lead to loss of supply to any customers), it is suggested that the System Minute indices be used.

For an event that produces an individual System Minute value that equals or exceeds one, this is characterized as a 'major event' and is reported separately and individually

\section{SYSTEM CHARACTERISTICS FOR BENCHMARKING}

\section{A. Harmonics}

For the purposes of benchmarking it is recommended that only voltage harmonics be considered. The justification for this is that current harmonic levels will be highly site specific and hence totally random. However, if similar allocation strategies are used and similar standards or regulations governing acceptable harmonic levels are in place, it is reasonable to assume that for sites with similar characteristics voltage harmonic levels should be comparable.

When the system characteristics which may impact harmonic performance are considered, there are a number of characteristics that will apply to all harmonic orders, while there are others which will be more applicable to either lower or higher order harmonics. For the purposes of the technical brochure, low order harmonics are defined as those less than or equal to the $15^{\text {th }}$, while high order harmonics are defined as those greater than the $15^{\text {th }}$.

The following are network characteristics which must be considered when benchmarking voltage harmonics:

- The harmonic allocation strategy, limits/planning levels and regulatory regime which is in place. A network operator will design and operate a network to suit the operating restrictions in place. There is no driver for a network to maintain harmonic levels below the limits which are in place. To do so, unnecessarily is an inefficient use of network capacity. Conversely where harmonic levels are being exceeded it may be necessary for network operators to install harmonic mitigation strategies to ensure compliance. For example, it is very difficult to compare networks which operate under IEEE harmonic limits with networks operating under IEC principles due to the fact that the different standards allow different voltage harmonic magnitudes.

- The network design/characteristics. Harmonic voltage levels are a function of the injected harmonic current and the network impedance. The design characteristics of the network will have significant impacts on the impedance of the network and hence harmonic voltage levels. network design characteristics that should be considered include:

-The voltage level of the network;

-The construction of the network, e.g. underground cable or overhead conductor;

-Whether the network is meshed or radial;

-The network security requirements. This determines the number of lines and transformers that must be kept in service;

-Known resonances;

-Known sources of harmonics such as load control ripple injections frequencies used at distribution voltage (also known as audio frequency injection control (AFIC)).

- The load/generation connected to the network. The loads/generators connected to the network determine the characteristics of the injected harmonic current. Different loads will have different harmonic current magnitudes as well as characteristic harmonic profiles.

\section{B. Flicker}

The most significant network parameter with regards to flicker is the system strength, usually expressed in terms of minimum and maximum short circuit capacity. Besides that, the information about large fluctuating installations like electric arc furnaces helps to perform a meaningful benchmarking of different systems. 


\section{Unbalance}

The level of voltage unbalance is influenced in general by the system strength. Other factors relevant are overhead line configuration, and type and connection of loads, e.g. traction load.

\section{Voltage Dips and Swells}

Characteristics of voltage dips and swells are dependent on short-circuits (e.g. dielectric breakdown due to atmospheric events, mechanical interference and damage, breakdown of network or user, accidents or errors in operation and maintenance), load fluctuations (e.g. starting of motors, connection or disconnection of reactive power compensation devices, gross fluctuation of loads), network (e.g. topology, type of neutral earthing, network design requirements (redundancy, protection), system strength (distribution of short-circuit power over the network, type of generation).

\section{E. Interruptions}

System characteristics important for benchmarking the interruption performance are:

- Circuit length proportion that is overhead line;

- Circuit length proportion that is cable;

- Redundancy of circuits and busbars;

- Lightning severity;

- Pollution severity;

- Failure rate of components.

Ideally the transmission networks benchmarked should be similar in the above respects.

\section{CONCLUSIONS}

This paper presented the results from the work of CIGRE WG C4.27 - Benchmarking of Power Quality Performance in Transmission Systems. The working group has addressed and analysed different aspects of PQ performance benchmarking in transmission system.

The paper presented the current understanding and an overview of international practice regarding power quality performance benchmarking. Thanks to a coordinated effort of many experts from around the world extensive data have been collected and the key features of existing practices extracted and reported.

The issues that need to be particularly addressed and for which a clear guidance should be provided include:

- The recommended indices or a set of indices, including "internal" indices;

- The subset of data to be used considering system characteristics;

- The benchmarking evaluation period;

- Consideration and management of data availability and sparse data;

- The statistical evaluation methods used to determine the indices for benchmarking for individual sites;

- The method by which individual site indices are combined to produce single indices for each data subset for large numbers of sites or entire networks.

The working group will continue to report on its activities regularly and to invite contributions from wide audience in order to provide as complete and practical guidelines for transmission system PQ performance benchmarking in current and future power networks.

\section{CIGRE WORKING GROUP C4.27 MEMBERS}

Working Group consists of 13 regular members from 10 different countries. Members: D. Vujatovic (UK, Convener), J. Kilter (EE, Secretary), S. Elphick (AU), Herwig Renner (AT), John van Coller (ZA), Frans van Erp (NL), Theo Laughner (US), Curtis Roe (US), Benjamin Genet (BE), Sergio Luis Pasero (ES), Peter Haigh (UK), Richard Ball (UK), Dalton Brasil (BR).

\section{REFERENCES}

[1] Power Quality Indices and Objectives. CIGRE Technical Brochure No 261, Working Group C4.07, 2004, 98 pp.

[2] J. Kilter, J. Meyer, B. Howe, F. Zavoda, L. Tenti, J. V. Milanovic, M. Bollen, P. F. Ribeiro, P. Doyle, and J. M. Romero Gordon, "Current Practice and Future Challenges for Power Quality Monitoring-CIGRE WG C4.112 Perspective". ICHQP 2012, 16-20 June, 2012, Hong Kong, China.

[3] IEC 61000-4-7: Electromagnetic compatibility (EMC) - Part 47: Testing and measurement techniques - General guide on harmonics and interharmonics measurements and instrumentation, for power supply systems and equipment connected thereto. IEC, 2009

[4] IEC 61000-4-30 (Ed. 3): Electromagnetic Compatibility (EMC) - Part 4-30: Testing and measurement techniques - Power quality measurement methods. IEC, 2015

[5] IEEE Std 519 (2014): IEEE Recommended Practice and Requirements for Harmonic Control in Electric Power Systems.

[6] IEC/TR 61000-3-6, (Ed. 2): Electromagnetic compatibility (EMC) - Part 3-6: Limits - Assessment of emission limits for the connection of distorting installations to MV, HV and EHV power systems. IEC, 2008.

[7] Engineering Recommendation G5/4-1 Planning Levels for Harmonic Voltage Distortion and The Connection of Non-Linear Equipment to Transmission Systems and Distribution Networks in The United Kingdom, October 2005.

[8] Standards Australia, TR IEC 61000.3.6:2012- Electromagnetic Compatibility (EMC) - Part 3.6: Limits-Assessment of emission limits for the connection of distorting installations to MV, HV and EHV power systems.

[9] CEER/ECRB, Guidelines of Good Practice on the Implementation and Use of Voltage Quality Monitoring Systems for Regulatory Purposes, 2012.

[10] IEC 61000-4-15: Electromagnetic compatibility (EMC) - Part 415: Testing and measurement techniques - Flickermeter - Functional and design specifications. IEC, 2010.

[11] IEEE Std 1453 (2015): IEEE Recommended Practice for the Analysis of Fluctuating Installations on Power Systems.

[12] CIGRE TB 449, Review of Flicker Objectives for LV, MV and HV Systems, WG C4.108, 2011.

[13] Problems of estimation technique for voltage fluctuation and research subjects - Comparison between $\triangle \mathrm{V} 10$ meter and IEC flicker meter - CRIEPI Report: T99041, 2000/05 (in Japanese).

[14] A. Novitskiy, H. Schau, "Analysis of a Ratio between $\Delta$ V10 and Pst Flicker Criteria", UPEC 2011, 46 ${ }^{\text {th }}$ International Universities' Power Engineering Conference, 5-8th September 2011, Soest, Germany.

[15] D. Arlt, M. Stark, and C. Eberlein, "Examples of International Flicker Requirements in High Voltage Networks and Real World Measurements", Proceedings of the 9th International Conference on Electrical Power Quality and Utilisation, Barcelona, Spain, Oct. 9-11, 2007.

[16] IEC/TR 61000-3-7 (Ed. 2): Electromagnetic compatibility (EMC) - Part 3-7: Limits - Assessment of emission limits for the connection of fluctuating installations to $\mathrm{MV}, \mathrm{HV}$ and $\mathrm{EHV}$ power systems. IEC, 2008.

[17] IEC 61000-3-13: Electromagnetic compatibility (EMC) - Part 3-13: Limits - Assessment of emission limits for the connection of unbalanced installations to MV, HV and EHV power systems. IEC, 2008. 